\title{
ON EVEN-TO-ODD MEAN LABELING OF SOME TREES
}

\author{
Leomarich F. Casinillo \\ Department of Mathematics and Physics \\ Visayas State University, Visca Baybay City, Leyte, Philippines \\ Email : leomarich_casinillo@yahoo.com
}

\begin{abstract}
Let $G=(V(G), E(G))$ be a connected graph with order $|V(G)|=p$ and size $|E(G)|=q$. A graph $G$ is said to be even-to-odd mean graph if there exists a bijection function $\varphi: V(G) \rightarrow\{2,4,8, \ldots, 2 p\}$ such that the induced mapping $\varphi^{*}: E(G) \rightarrow$ $\{3,5, \ldots,(2 p-1)\}$ defined by $\varphi^{*}(u v)=\frac{\varphi(u)+\varphi(v)}{2} \forall u v \in E(G)$ is also bijective. The function $\varphi$ is called an even-to-odd mean labeling of graph $G$. This paper aimed to introduce a new technique in graph labeling called even-to-odd mean labeling. Hence, the concepts of even-to-odd mean labeling has been evaluated for some trees. In addition, we examined some properties of tree graphs that admits even-to-odd mean labeling and discussed some important results.
\end{abstract}

Keywords: Trees, even-to-odd mean labeling, even-to-odd mean graph

\section{INTRODUCTION}

In Graph theory, labeling in graphs remain intriguing and has undergone tremendous development through research. The first and most famous labeling in graphs is graceful labeling [1]. At present, there are a lot graph theorist who contributed to the development of labeling in graphs (e.g. [2-5]). A detailed survey in graph labeling is found in the paper of [1]. Before we introduce the concept of labeling, we first need the following definition of terms. Connected graph $G$ is a pair of vertex set $V(G)$ and edges set $E(G)$. The cardinality $|V(G)|$ of $V(G)$ is the order of $G$ and the cardinality $|E(G)|$ of $E(G)$ is the size of $G$. A walk is a sequence $u_{1}, u_{2}, \ldots, u_{n}$ of vertices of graph $G$ such that $\left\{u_{i}, u_{i+1}\right\} \in E\left(P_{n}\right)$ for each $i=1,2, \ldots, n$. Vertices $u_{1}$ and $u_{n}$ are the endpoints of the walk while the vertices $u_{2}, u_{3}, \ldots, u_{n-1}$ are internal vertices of the walk. The length of walk is the number of edges on the walk, i.e., the walk $u_{1}, u_{2}, \ldots, u_{n}$ has length $n-1$. A path is a walk that does not repeat edges and does not end where it starts, i.e., $u_{1} \rightarrow u_{2} \rightarrow \ldots \rightarrow u_{n}, u_{1} \neq u_{n}$. A path of order $n$ and length $n-1$ is denoted by $P_{n}$ where $n \geq 1$. A cycle is a walk that does not repeat edges and does end where it starts, i.e., $u_{1} \rightarrow u_{2} \rightarrow \ldots \rightarrow u_{n} \rightarrow u_{1}$. A cycle graph of order $n$ and length $n$ is denoted by $C_{n}$ where $n \geq 3$. A tree is a connected acyclic graph or a connected graph with no cycles. A tree with $n$ vertices has $n-1$ edges. The edges of a tree are known as branches, and the elements of a tree are called nodes or vertices.

For other concepts of graph theory, readers may refer to the following references [7-13]. We also introduce a new graph in this paper, that is, the $\mathrm{H}$ graph. $\mathrm{H}$ graph is a tree that compose of two paths $P_{n}$ and $P_{m}$ with odd order greater or equal to 3.The two paths is connected by an edge in the middle vertex of paths $P_{n}$ and $P_{m}$. Hence, the order of $\mathrm{H}$ graph is $m+n$; and $\mathrm{H}$ graph is denoted by $G=P_{n} \leftrightarrow P_{m}$. See the figure below. 


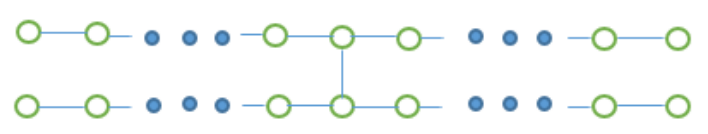

Figure 1. Graph $G=P_{n} \leftrightarrow P_{m}$.

In the paper of Manickam and Marudai [3], the concept of odd mean labeling of graph is introduced. A graph $G$ is said to be odd mean if there exists an injective function $f: V(G) \rightarrow$ $\{1,3, \ldots, 2 q-1\}$ defined by $f^{*}(u v)=\left|\frac{f(u)+f(v)}{2}\right|$ is a bijection. On the other hand, the concept of even mean labeling was introduced by Gayathri and Gopi [2]. A graph $G$ is said to be even mean if there exists an injective function $g: V(G) \rightarrow\{2,4, \ldots, 2 q\}$ defined by $g^{*}(u v)=$ $\left|\frac{f(u)+f(v)}{2}\right|$ is a bijection. Motivated by these labeling concepts, our effort focused on introducing new concept called even-to-odd mean labeling in graphs. A graph $G=$ $(V(G), E(G))$ is said to be even-to-odd mean graph if there exists a bijection function $\varphi$ : $V(G) \rightarrow\{2,4,8, \ldots, 2 p\}$ such that the induced mapping $\varphi^{*}: E(G) \rightarrow\{3,5, \ldots,(2 p-1)\}$ defined by $\varphi^{*}(u v)=\frac{\varphi(u)+\varphi(v)}{2} \forall u v \in E(G)$ is also bijective. The function $\varphi$ is called an even-to-odd mean labeling of graph $G$. This new technique is stricter compared to existing labeling techniques in literature. In this paper the concepts of even-to-odd mean labeling has been evaluated for some trees. Furthermore, we examined some properties of tree graphs that admits even-to-odd mean labeling..

\section{METHODOLOGY}

In this paper, we follow the methodology of the current paper of [14] that is exploratory in nature. Firstly, we introduced and evaluated a new concept of labeling in graphs, that is, evento-odd mean labeling. Secondly, we examined some trees that admits even-to-odd mean labeling. Furthermore, we disproved that graphs that contain a cycle does not admits even-toodd mean labeling and provided a conjecture that some trees can be constructed satisfying the condition of even-to-odd mean labeling.

\section{RESULTS}

The following result is a direct consequence of the definition of even-to-odd mean labeling showing that a path $P_{n}$ where $n \geq 2$ admits even-to-odd mean labeling.

Theorem 1. If $G=P_{n}$ where $n \geq 2$, then $G$ is an even-to-odd mean graph.

Proof. Suppose that $G=P_{n}$ where $n \geq 2$. Then, we have $V(G)=\left\{u_{i} \mid 1 \leq i \leq n\right\}$ and $E(G)=$ $\left\{u_{i-1} u_{i} \mid 2 \leq i \leq n\right\}$. Clearly, we obtain $|V(G)|=n$ and $|E(G)|=n-1$. Now, we let $\varphi$ : $V(G) \rightarrow\{2,4,8, \ldots, 2 n\}$. Then, we define the following: $\varphi\left(u_{j-1}\right)=2 j-2$ where $2 \leq j \leq$ $n+1$ and $\varphi\left(u_{j}\right)=2 j$ where $1 \leq j \leq n$. Then, it follows that $\varphi^{*}\left(u_{j-1} u_{j}\right)=2 j-1$ where $2 \leq j \leq n$ and induced a mapping $\varphi^{*}: E(G) \rightarrow\{3,5, \ldots,(2 n-1)\}$. Hence, $G$ admits even-toodd mean labeling and this completes the proof.

Illustration. Consider even-to-odd mean labeling of path $P_{5}$. 


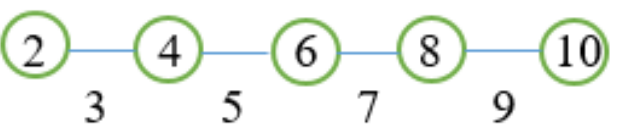

Figure 2. Even-to-odd mean labeling of $P_{5}$.

The following corollaries are quick from Theorem 1 showing the value of the bijection function $\varphi$ and $\varphi^{*}$.

Corollary 2. Let $G=P_{n}$ where $n \geq 3$. If $n \equiv 1(\bmod 2)$, then $\varphi\left(\frac{u_{n+1}}{2}\right)=n+1$.

Corollary 3. Let $G=P_{n}$ where $n \geq 2$. If $n \equiv 0(\bmod 2)$, then $\varphi^{*}\left(u_{\frac{n}{2}} v_{\frac{n+1}{2}}\right)=n+1$.

Now, if we let $\overline{\varphi(u)_{4}}=\lambda$ where $\varphi(u) \equiv \lambda(\bmod 4) \forall u \in V(G)$ where $G=P_{n}$, then the following theorem is obtain.

Theorem 4. Let $G=P_{n}$ and $J=P_{n+1}$ where $n \geq 2$. Then, the following holds:

$$
\text { i. } \sum_{i=1}^{n} \overline{\varphi\left(u_{l}\right)_{4}}=\sum_{i=1}^{n+1} \overline{\varphi\left(v_{J}\right)_{4}}=n+1 \quad \text { where } n \equiv 1(\bmod 2)
$$

and

$$
\text { ii. } \sum_{i=1}^{n} \overline{\varphi\left(u_{l}\right)_{4}}=\sum_{i=1}^{n+1} \overline{\varphi\left(v_{J}\right)_{4}}=n \quad \text { where } n \equiv 0(\bmod 2)
$$

where $u_{i} \in V(G)$ and $v_{j} \in V(J)$.

Proof. Suppose that $G=P_{n}$ and $J=P_{n+1}$ where $n \geq 2$. Then, by Theorem 1, graph $G$ and $J$ admits even-to-odd mean labeling. And we consider the two cases below:

Case 1 . Suppose that $n \equiv 1(\bmod 2)$, it is clear that there are $\frac{n+1}{2}$ of $\overline{\varphi(u)_{4}}=2=\overline{\varphi(v)_{4}}$, where $u \in V(G)$ and $v \in V(J)$. Hence, we obtain

$$
\sum_{i=1}^{n} \overline{\varphi\left(u_{\imath}\right)_{4}}=\sum_{i=1}^{n+1} \overline{\varphi\left(v_{J}\right)_{4}}=2\left(\frac{n+1}{2}\right)=n+1
$$

where $u_{i} \in V(G)$ and $v_{j} \in V(J)$.

Case 2. Suppose that $n \equiv 0(\bmod 2)$, it is easy to check that there are $\frac{n}{2}$ of $\overline{\varphi(u)_{4}}=2=\overline{\varphi(v)_{4}}$, where $u \in V(G)$ and $v \in V(J)$. Hence, we obtain

$$
\sum_{i=1}^{n} \overline{\varphi\left(u_{\imath}\right)_{4}}=\sum_{i=1}^{n+1} \overline{\varphi\left(v_{\jmath}\right)_{4}}=2\left(\frac{n}{2}\right)=n
$$

where $u_{i} \in V(G)$ and $v_{j} \in V(J)$. And this completes the proof.

Remark 5. Let $u, v \in V(G)$ where $u v \in E(G)$. Then, either one of the following holds true:

i. If $\varphi(u) \equiv 0(\bmod 4)$, then $\varphi(v) \equiv 2(\bmod 4) ;$ or

ii. If $\varphi(v) \equiv 0(\bmod 4)$, then $\varphi(u) \equiv 2(\bmod 4)$.

The next Theorem below shows that any $\mathrm{H}$ graph admits the even-to-odd mean labeling. 
Theorem 6. Any H graph admits an even-to-odd mean labeling.

Proof. Suppose that $G=P_{n} \leftrightarrow P_{m}$ is an $\mathrm{H}$ graph. Then, we consider $P_{n}$ as the first path of $G$ and $P_{m}$ is the second path of $G$ to have odd order of $m=n>3$ and it follows that the order of $G$ is $n+m$. Let $\varphi: V(G) \rightarrow\{2,4,8, \ldots, 2(n+m)\}$. Hence, for the first path $P_{n}$, we define $\varphi\left(u_{i-1}\right)=2 i-1$ where $2 \leq i \leq n+1$ and $\varphi\left(u_{i}\right)=2 i$ where $1 \leq i \leq n$. On the other hand, for the second path $P_{m}$, we also define $\varphi\left(u_{j-1}\right)=2 j-1$ where $n+2 \leq i \leq n+m+1$ and $\varphi\left(u_{j}\right)=2 j$ where $n+1 \leq i \leq n+m$. So, it implies that $\varphi^{*}\left(u_{j-1} u_{j}\right)=2 j-1$ where $2 \leq$ $j \leq n$ and $\varphi^{*}\left(u_{i-1} u_{i}\right)=2 i-1$ where $n+2 \leq j \leq n+m$. In addition, since $n+m$ is even, we have $\overline{\varphi\left(\frac{\left.u_{n+1}\right)_{4}}{2}\right.}=2$ and $\overline{\varphi\left(\frac{\left.v_{m+1}\right)_{4}}{2}\right.}=0$, or $\overline{\varphi\left(\frac{\left.u_{n+1}\right)_{4}}{2}\right.}=0$ and $\overline{\varphi\left(\frac{v_{m+1}}{2}\right)_{4}}=2$. Hence, $\varphi^{*}\left(u_{\frac{n+1}{2}} v_{\frac{m+1}{2}}\right) \equiv 1(\bmod 2)$ and induced a mapping $\varphi^{*}: E(G) \rightarrow\{3,5, \ldots,(2(n+m)-1)\}$. And this completes the proof.

Illustration. Consider even-to-odd mean labeling of $\mathrm{H}$ graph $P_{3} \leftrightarrow P_{3}$.

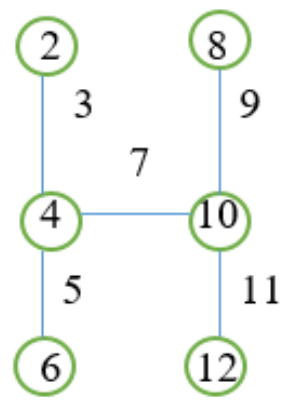

Figure 3. Even-to-odd mean labeling of $P_{3} \leftrightarrow P_{3}$.

Corollary 7 below is immediate from Theorem 6 showing the value of induced mapping $\varphi^{*}$ in $\mathrm{H}$ graph.

Corollary 7. Let $G=P_{n} \leftrightarrow P_{m}$. For any $m=n$ (odd integers), we have $\varphi^{*}\left(\frac{u_{\frac{n+1}{2}} v_{\frac{m+1}{2}}}{2}\right)=n+$ $m+1$.

The next results shows that a cycle graph and graph that contain cycles does not admits even-to-odd mean labeling.

Theorem 8. Let $G=C_{n}$ where $n \geq 3$. Then, $G$ is not an even-to-odd mean graph.

Proof. Suppose that $G=C_{n}$ where $n \geq 3$. Then, we let $\left\{u_{1}, u_{2}, \ldots, u_{n}\right\}$ be the set of vertices of $G$ and $\left\{u_{1} u_{2}, u_{2} u_{3}, \ldots, u_{n-1} u_{n}, u_{n} u_{1}\right\}$ be the set of edges in $G$. This simply follows that $|V(G)|=n$ and $|E(G)|=n$. Let $\varphi: V(G) \rightarrow\{2,4,8, \ldots, 2 n\}$. Then, consider the following cases below:

Case 1 . Consider $n \equiv 1(\bmod 2)$ and define the following: $\varphi\left(u_{j-1}\right)=2 j-2$ where $2 \leq j \leq$ $n$ and $\varphi\left(u_{j}\right)=2 j$ where $2 \leq j \leq n$. Then, it follows that $\varphi^{*}\left(u_{j-1} u_{j}\right)=2 j-1$ where $2 \leq$ $j \leq n$. However, if $\varphi\left(u_{1}\right) \equiv 2(\bmod 4)$, then, $\varphi\left(u_{n}\right) \equiv 2(\bmod 4), \quad$ or if $\varphi\left(u_{1}\right) \equiv$ 
$0(\bmod 4)$, then, $\varphi\left(u_{n}\right) \equiv 0(\bmod 4)$. This does not induced a mapping $\varphi^{*}: E(G) \rightarrow$ $\{3,5, \ldots,(2 p-1)\}$.

Case 2 . Consider $n \equiv 0(\bmod 2)$. Again, we define the following: $\varphi\left(u_{i-1}\right)=2 i-2$ where $2 \leq i \leq n$ and $\varphi\left(u_{i}\right)=2 i$ where $2 \leq i \leq n$. Then, it implies that $\varphi^{*}\left(u_{i-1} u_{i}\right)=2 i-1$ where $2 \leq i \leq n$. Now, if $\varphi\left(u_{1}\right) \equiv 2(\bmod 4)$, then, $\varphi\left(u_{n}\right) \equiv 0(\bmod 4), \quad$ or if $\varphi\left(u_{1}\right) \equiv$ $0(\bmod 4)$, then, $\varphi\left(u_{n}\right) \equiv 2(\bmod 4)$. However, $\varphi^{*}\left(u_{1} u_{n}\right)=\mu$ and $2 \leq \mu \leq 2 i-1$ where $1 \leq i \leq n$. This contradicts to the bijectivity of the induced mapping of even-to-odd mean labeling.

Hence, $G$ is not an even-to-odd mean graph. And this completes the proof.

Corollary 9. Let $G$ be a graph that contains a cycle/s. Then, $G$ is not an even-to-odd mean graph.

Proof. Immediate from Theorem 8.

In view of Theorem 8, we arrived an assumption that even-to-odd mean labeling only works for tree graphs including paths and $\mathrm{H}$ graphs. Hence, the following conjecture is constructed.

Conjecture 10. Let $k$ be a positive integer. If graph $G$ with order $n \geq 7$ admits even-to-odd mean labeling, then there exists $k$ trees that can be form such that $n<k$.

\section{CONCLUSION}

It is concluded that even-to-odd mean labeling is stricter compared to existing labeling techniques in literature since there is only one possible bijection in the induced mapping, that is, all elements in the domain and co-domain of the function $\varphi$ are all used up. Results revealed that paths and $H$ graphs admits the condition of even-to-odd mean labeling. We had also generated some new results regarding the properties of path and $\mathrm{H}$ graphs satisfying even-toodd mean labeling. In addition, we had shown that a cycle graphs and any graphs that contain cycle/s does not admits even-to-odd mean labeling.

For future research, one may consider the number of ways in labeling path and $H$ graphs satisfying even-to-odd mean labeling. Furthermore, it would be interesting constructing $k$ trees satisfying the conditions of even-to-odd mean labeling in a graph $G$ with order $n \geq 7$ such that $n<k$.

\section{REFERENCES}

[1] Gallian, J.A., "A dynamic survey of graph labeling," The Electronic Journal of Combinatorics, vol. 18, 2014.

[2] Gayathri, B. \& Gopi, R., "Cycle related mean graphs," Elixir International Journal of Applied Sciences, vol. 71, pp. 25116-25124, 2014.

[3] Manickam, K. \& Marudai, M., "Odd mean Labeling of graphs," Bulletin of Pure and Applied Sciences, vol. 25, no. 1, pp. 149-153, 2006.

[4] Ponraj, R. \& Somasundaram, S., "Mean Labeling of graphs," National Academy Science Letter, vol. 26, pp. 210-213, 2003. 
[5] Vasuki, R., Nagarajan, A. \& Arockiaraj, S., "Even vertex odd mean labeling of graphs," Sut. J. Math, vol. 49, no. 2, pp. 79-92, 2013.

[6] Bollobás, B., Modern graph theory, New York : Springer-Verlag, 1998.

[7] Casinillo, L. F., "A note on Fibonacci and Lucas number of domination in path," Electronic Journal of Graph Theory and Applications, vol. 6, no. 2, pp. 317-325, 2018.

[8] Casinillo, L. F., "Odd and even repetition sequences of independent domination number," Notes on Number Theory and Discrete Mathematics, vol. 26, no. 1, pp. 8-20, 2020.

[9] Chartrand, G. and Zhang, P., A First Course in Graph Theory, New York: Dover Publication, Inc., 2012.

[10] Kumar, M. K., Murali, R. \& Chidanandan, V., "Domination in some classes of ditrees," Bulletin of the International Mathematical Virtual Institute, vol. 6, pp. 157-167, 2016.

[11] Ore, O., Theory of Graphs, American Mathematical Society Provedence, 1962.

[12] Varkey, M. T. K. \& Thomas, M. S., "Some tree and tree related cubic graceful graphs," International Journal of Advanced Scientific and Technical Research, vol. 7, no. 4, pp. 239-244, 2017.

[13] Casinillo, L. F., Lagumbay, E. T. \& Abad, H. R. F., A note on connected interior domination in join and corona of two graphs," IOSR Journal of Mathematics, vol. 13, no. 2, pp. 66-69, 2017.

[14] Casinillo, L. F., "Some new notes on Mersenne primes and perfect numbers," Indonesian Journal of Mathematics Education, vol. 3, no. 1, pp. 15-22, 2020. 